\title{
Research on Cross-Regional Emergency Scheduling and Allocating Strategies
}

\author{
Fan Zhang ${ }^{1}$, Yuan $\mathrm{Gao}^{2}$ and Yueli $\mathrm{Li}^{3}$ \\ ${ }^{1-3}$ School of Information Science and Technology, Agricultural University of Hebei, \\ No.289,Lingyusi Street, Baoding 071001, P.R.China \\ 1ellenzhang0911@126.com, ${ }^{2}$ gaoyuan_83_91@126.com, ${ }^{3}$ li_yueli@163.com
}

\begin{abstract}
Aiming at the present situation of lacking of scientific and reasonable schemes in farm machinery emergency scheduling and allocating, farm machinery emergency scheduling and allocating mode leading by management department is proposed and the optimization model with the goal of cost as low as possible and completing emergency tasks within the prescribed period is established in the paper. According to the characteristics of farm machinery emergency scheduling and allocating, the scheduling and allocating algorithm with priority rules and the scheduling and allocating algorithm based on the non-cooperative game theory are proposed. Compared with two algorithms, the scheduling cost and the allocating amounts calculated by the algorithm based on the non-cooperative game theory are less than that of the algorithm with priority rules. It is concluded that the scheduling and allocating algorithm based on the non-cooperative game theory in the paper is more effective and reasonable.
\end{abstract}

Keywords: Emergency Scheduling and Allocating, Dynamic Priority Rules, Scheduling Cost, Allocating Strategies

\section{Introduction}

Reasonable scheduling and allocating strategies play a very important role in the successful completion of emergency reaping or planting tasks. When the emergency tasks are cleared, the recent farm machinery are scheduled and allocated. However, there are some problems in the farm machinery emergency service. Firstly, the agricultural management allocates the farm machinery by artificially or empirically. It often happened that farm machinery crowds in some regions and there is not enough machinery to complete the harvest in other regions. So it can't guarantee the entire allocating plan reasonable. Secondly, farm machinery from one of owners usually can't complete an emergency service task, farm machinery should be scheduled from more than one owners. Thirdly, the characteristics of emergency scheduling and allocating is urgent, if there is no reasonable allocating plan, the cost will be increased. Therefore, farm machinery scheduling and allocating strategies are needed in this situation ${ }^{[1-2]}$. The research of farm machinery scheduling and allocating problem has important practical and theoretical significance.

Multi-machine scheduling problem with the concept of evolutionary game and Nash equilibrium are studied in the paper ${ }^{[3]}$. The work pieces are considered as the game players, the machines are considered as the strategy set. The game Nash equilibrium solution is be calculated by the payment matrix, which is obtained through choosing the different machines to construct the work piece operation. The task scheduling algorithm based on Nash equilibrium is studied in this paper ${ }^{[4]}$. Considering the performance of the resource and network, each scheduling node is considered as a game player and how to maximize the average task completion time is as the target. The Nash equilibrium is a 
hybrid strategy for each scheduling node. The superiority of the proposed scheduling algorithm is verified through simulation. The emergency resource scheduling model and algorithm based on non-cooperative game are proposed from the point of view of multi disaster point in the paper ${ }^{[5]}$. The various disaster points are mapped into the game model and the possible resource scheduling scheme is mapped to the strategy set. The reciprocal of the resource scheduling cost is a utility function and the emergency resource scheduling problem is transformed into the Nash of the non-cooperative game scheduling model in this scheduling model. Then, an iterative algorithm for solving the Nash equilibrium solution is presented. Finally, the validity and feasibility of the model are verified by simulation test. The grid resource allocation strategy based on sequential game theory is proposed in the paper ${ }^{[6]}$. The problem of multiple users competing with the same computing resource is converted into the problem of multi-users sequential game. The strategy is to predict the resource load by using the Nash equilibrium solution of each stage game in the sequential game theory. Then the load information is made to generate the optimal bid price for all the users and resources. The problem of allocating the bandwidth of wireless network is constructed to a non -cooperative game system by the formula ${ }^{[7]}$. The simulation results show that the wireless resource allocation mechanism based on the non-cooperative game theory can effectively solve the problem of the allocation of the bandwidth and the number of connections, and can guarantee the rationality and fairness of the allocation. The game theory is applied to the research of network resource allocation in this paper. the method of real-time queue management based on game theory the method of real-time queue management based on game theory and the equilibrium routing algorithm based on non-cooperative game theory is proposed and simulation results of the algorithm is verified effectively. ${ }^{[8]}$ The single machine scheduling of non-cooperative game model is given and the existence of Nash equilibrium solution is discussed in the paper ${ }^{[9]}$. Considering that different work pieces with different optimization objectives, a relaxation iterative algorithm for solving the Nash equilibrium solution with multi-objective multi-users are designed. The experimental results show that the scheduling results well balance the interests of all players. The game scheduling problem is a typical resource allocation and competition problem with the constrained and optimized index requirements. Because of the scarcity of resources, the individual interests of participating in the distribution of resources may be inconsistent. Therefore conflicts are existed in the scheduling problem. Game theory can be a good method to analyze the scheduling problem ${ }^{[10-17]}$.

In order to solve the existed emergency scheduling and allocating problems, a scientific and rational allocating scheme is proposed in the paper. Farm machinery emergency scheduling and allocating models are established with the goal of low scheduling cost.

\section{Problem Statement and Preliminaries}

When emergency tasks are cleared, farm machinery management department collect the information about emergency farmland position, emergency response time, farm machinery service capability and so on. Grasping the information of farmland and farm machinery timely and accurately plays a very important role for analysis of emergency scheduling and allocating strategies. The targets of emergency scheduling and allocating are that completing the emergency tasks within the prescribed period and the scheduling and allocating cost being as low as possible under the competition of the emergency task points for farm machinery.

To facilitate the description of the problem, the farmland is called as tasks and the farm machinery is called as resources in the paper. The emergency scheduling and allocating problem with many impact factors is complex. The related variables are described and several concepts are explained in the following, in order to deeply study the problem. 
$M_{i}$ means the resource point $i$, and $i=1,2, \ldots m$.

$S_{j}$ means the area of the emergency task $j, j=1,2, \ldots n$.

$t_{i j}$ means the scheduling time from $i$ to $j$.

$s t_{i}$ is the response time of farm machinery point $i$.

$a t_{i j}$ is the arrival time from $i$ to $j$.

$T_{j}$ is the completion time of farmland $j$.

$r$ means service capability of unit time.

$d$ is the cost for unit of scheduling time.

$W_{i j}$ is the traffic condition from $i$ to $j$.

$V_{i j}$ is average speed from $i$ to $j$ in the ideal condition.

$n_{i j}$ is the amount of farm machinery scheduled from $i$ to $j, n_{i j}>=0$.

$T_{\text {deadline }}$ means the prescribed time of emergency scheduling and allocating.

The information of the resources mainly includes the location of the resources, amounts of the resources, the response time of resources and the operational capability of every resource.

The response time means the time that resources begin to participate in the emergency tasks. The farm machinery scheduling and allocating time consists of two parts in the paper. One is the transiting time of farm machinery. The other is the time that farm machinery serves for farmland. So the completion time is equal to the response time and transportation time and service time.

Transportation time means the time transporting from the location of resources to the location of the emergency tasks or other resources. It relates to the distance and farm machinery transport average speed. The average speed is related to road condition. $W_{\mathrm{ij}}$ means the traffic conditions from $i$ to $j$, with which a number between $[0,1]$ to represent.

The larger $W_{\mathrm{ij}}$ is, the better road traffic condition is. $V_{i j}^{\prime}$ means actual transporting speed from $i$ to $j$, it can be defined as:

$V_{i j}^{\prime}=V_{i j} * W_{i j}$

So the scheduling and allocating time from $i$ to $j$ can be defined as:

$t_{i j}=\frac{D_{i j}}{V_{i j}^{\prime}}$

With the goal of the emergency scheduling and allocating and the above analysis, the model is established as follows.

$\min d \sum_{i=1}^{m} \sum_{j=1}^{n} t_{i j} n_{i j}$

St.

$T_{j} \leq T_{\text {deadline }} \quad j=1,2, \cdots, n$

$t_{i j}=\frac{D_{i j}}{V_{i j} * W}$

$s t_{i}+t_{i j}=a t_{i j} \quad i=1,2, \cdots, m \quad j=1,2, \cdots, n$

$a t_{i j}<T_{\text {deadline }} \quad i=1,2, \cdots, m \quad j=1,2, \cdots, n$

$\mathrm{r} \sum_{i=1}^{m} \sum_{\mathrm{j}=1}^{\mathrm{n}}\left(\mathrm{T}_{\mathrm{j}}-a t_{i j}\right) \mathrm{n}_{\mathrm{ij}}=S_{j}$

From the constraint condition (4) can see, the completion time of task $j$ should be no later than $T_{\text {deadline. }}$. The constraint condition (5) shows that the transportation time from $i$ to $j$ is related to road and weather condition. The constraint condition (6) shows that the arrival time from $i$ to $j$ equals the sum of the response time $s t_{i}$ of $i$ and the transportation time from $i$ to $j$. From the constraint condition (7) can see, the arrival time that from $i$ to $j$ 
should be no later than $T_{\text {deadline. }}$ The constraint condition (8) means the total completion areas equals the areas of all the tasks.

From the above discussion can see, when the emergency events occurs, the suitable allocating strategies of farm machinery are very important for each farmland to complete the emergency task as soon as possible. When more than one farmland needs the service of farm machinery at the same time, farm machinery should be scheduled and allocated in the certain order. The scheduling and allocating order will be studied in the paper. The sooner the emergency completion time is, the better is in the general emergency scheduling and allocating. All the farmland is expected to be complete before the deadline time.

\section{Emergency Scheduling and Allocating Algorithm based on Priority Rules}

The emergency scheduling and allocating is a complex decision problem, which mainly include two aspects of the decision-making. The first one is to make decision how to choose the emergency task. The second one is to make decision how to schedule and allocate for every emergency task. The emergency scheduling and allocating algorithm based on priority rules is proposed in the paper. The emergency scheduling and allocating schemes of every task with low-cost are obtained firstly, and the schemes with conflict are adjusted based on priority until all the tasks are allocated before the deadline. The steps of the emergency scheduling and allocating are divided into the following parts.

Summarized information of tasks and resources. The amounts of the emergency resources should be approximately calculated when the emergency events have occurred. If the total number of existing agricultural resources is less than the needed amount of tasks, it is shortage of resources. Therefore, the agricultural resources should be added until the available resources are not less than the sum of the demand so far.

Independent optimal solution. The emergency scheduling and allocating target is to complete the farmland tasks within the deadline time and the emergency scheduling and allocating costs as small as possible. The more the amount of resources are, the greater the scheduling and allocating costs are. The longer the distance of scheduling and allocating are, the greater the costs are. Therefore, the minimum distribution and recently priority allocating strategies are proposed to obtain the independent optimal scheme is the temporal distance matrix, which is among every task and every resource. $T=\left(t_{i j}\right)_{i} \times_{j}, t_{i j}$ is the scheduling time from $i$ to $j$. If $s t_{i}+t_{i j} \geqslant T_{\text {deadline, }}$, it means the resource $i$ can't finish the task $j$.

1. Minimum distribution rule. The farmland will be assigned the minimum amounts of farm machinery that can complete the task within the deadline time.

2. Recently priority in the allocating rule. The nearest farm machinery will be first to allocate for the farmland until the completion of the task of this farmland.

Different resources may have different emergency response time. So the emergency response time will be unified for the following research. $T_{\text {current }}$ is the current time, $T_{\text {deadline }}$ is the deadline time. $T_{\text {response }}^{i}$ is the response time of the task $i . t_{i j}$ is transferring time from $i$ to $j$. the period time of $\left(T_{\text {response }}^{i}-T_{\text {current }}\right)$ is as a part of the transferring time, so the transferring time from $i$ to $j$ is equal to $t_{i j}+\left(T_{\text {response }}^{i}-T_{\text {current }}\right)$. Meanwhile, the scheduling cost from $i$ to $j$ will be increased. The treatment is more reasonable in the real situation. If the resource $i$ is near to the task $j$, but the response time of $i$ is later than that of other's, so the resource $i$ is not the best choice for solving the independent optimal scheme.

Tasks sorting based on priority. How to obtain a reasonable scheduling and allocating scheme for the farmland is a complex issue when the emergency events 
happened. The emergency order is decided by the priority of the tasks, the higher the priority, the earlier the task is be scheduled and allocated. The priority of the tasks is studied in the following.

The priority of tasks is considered at the aspects of important grade and the minimum demand amounts. The important grade of tasks is mainly from the evaluation of the farm machinery management department. The department will classify the tasks for different grades. The higher grade of tasks will have a high priority to schedule and allocate.

The target of emergency scheduling and allocating is that the completing time of all the emergency tasks is as short as possible. So the minimum requirement of every task is analyzed per unit time in the paper, which is denoted as $N^{\mathrm{min}}$.

$N_{j}^{\min }=\frac{\sum_{i=1}^{m} n_{i j}\left(T_{\text {deadline }}-T_{\text {response }}^{i}\right)}{T_{\text {deadline }}-T_{\text {response }}^{\min }}$

$N^{m i n}{ }_{j}$ is the minimum requirement of the task $j . n_{i j}$ is the number of resources $i$ allocated for the task $j$.

$T_{\text {response }}^{i}$ is the response start time of the resource $i . T_{\text {response }}^{\min }$ is the earliest response time of the task $j$.

$E P_{j}=\sum_{i=1}^{2} \omega_{i} \beta_{i}$

$E P_{j}$ is the priority of the task $j . \beta_{i}$ is the evaluation value of $i^{\text {th }}$ influence factors. $\omega_{\mathrm{i}}$ is the weights of $i^{\text {th }}$ influence factors. $\beta_{1}$ is the evaluation value of importance of tasks, $\beta_{2}$ is the evaluation value of the minimum requirement of every task in unit time. $\omega_{\mathrm{i}}$ is obtained by fuzzy analytic hierarchy process.

Conflict detection and treatment. There may be conflicts among the independent optimal schemes. If there is conflict, the resources which are unallocated will be reassigned for the task.

According to the above analysis, two steps of emergency scheduling and allocating based on Priority Rules. Firstly, the steps of the independent optimal scheme of every task are designed in this paper.

Step 1 Initialization

Step 1.1 Create queue $Q$, pre-allocating table $P T$.

Step 1.2 Sort the tasks according to the scheduling and allocating costs from low to high, and save in $Q$.

Step $1.3 \mathrm{k}=1$.

Step 2 If $Q$ is null ,turn to Step 5.

Step3 Remove $k^{\text {th }}$ resource from $Q, n_{k}$ is the allowable number of resource $k$. If $r n_{k}\left(T_{\text {deadline }}-T_{\text {response }}^{k}\right) \geq S_{j}$, then turn to Step5.

Step $4 S_{j}=S_{j}-r n_{k}\left(T_{\text {deadline }}-T_{\text {response }}^{k}\right)$, save the allocating scheme in $P T, k++$, turn to Step 3.

Step5 Quit.

Secondly, the emergency scheduling and allocating algorithm based on priority is designed after the independent optimal scheme is calculated. The steps are as follows.

Step1. Initialization.

Step 1.1 Create tasks pre-allocating table $P T$, tasks queue $Q$, idle resource queue $F Q$. 

task.

Step 1.2 Calculate the optimal emergency scheduling and allocating scheme of each

Step 1.3 Sort the tasks based on priority.

Step $1.4 s=1$.

Step 2. If $Q$ is null, turn to Step5.

Step 3. Remove $s^{\text {th }}$ resource from $Q$, if no conflict in the allocating scheme of $s^{\text {th }}$ resource, $s++$, turn to Step2.

Step 4. Allocate resources for $s^{\text {th }}$ task. The location of the resources is nearest to $s^{\text {th }}$ task's and the amount of them can meet the minimal demand, $s++$,turn to Step2.

Step5. Exit.

\section{Emergency Scheduling and Allocating Algorithm based on the Non-Cooperative Game Theory}

The demand of each farmland for farm machinery is in conflict in farm machinery emergency scheduling. In order to reconcile these conflicts, the contradiction, which is among the competition of farmland for farm machinery, should be resolved. Game theory is the subject that research on resolving and reconciling the conflicts. According to game theory, the decision makers is as an outsider, the various farmland operations points are as the players, and the value of farmland operations points in a scheme is as payment of players in one condition. Therefore, farm machinery scheduling and allocating schemes is the game theory strategy. Once the target condition is determined, the strategy sets also will be determined. Meanwhile corresponding payment will be produced. The solutions of game, which are corresponding to decision problem of farmland operational points, are obtained by game selection. Decision makers need to select satisfactory solution from solutions of game based on their preferences and utility. Farm machinery emergency scheduling problem is linked to game theory together theoretically.

(1) The basic information of farm machinery and emergency farmland are summarized and emergency farm machinery is sorted by response time. The total amounts of the farm machinery that needed by every farmland are calculated and compared with the total number of farm machinery that can be available by the emergency point. If the total demand amounts are greater than the total supplied, the next emergency agricultural point should be added, until the total demand amounts is no more than the total supplied amounts.

(2) Independent optimal scheme of every agricultural operations are solved by allocating strategies of the minimum requirements and the recent priority rules.

(3) Judging if the conflict is among each emergency agricultural points by the order of responses time. If the farm machinery of the emergency points can meet the distribution results, then farm machinery is scheduled and allocated by

(4) The multi-point emergency deployment of agricultural operations game model is established and the Nash equilibrium should be solved, then the agricultural operational points will be scheduled and allocated by the Nash equilibrium.

(5) If the emergency tasks of agricultural points don't complete by Nash equilibrium, then the recent farm machinery will be allocated until the tasks of these agricultural operational points can be completed.

According to the above analysis, four solving steps of emergency scheduling and allocating based on the non-cooperative game theory, are as follows.

Step1. The initialization of agricultural pre-allocation process 
Step 1.1 Sort the farm machinery for every farmland according to the scheduling and allocating costs from low to high.

Step 1.2 calculate the allocating scheme of every farmland operational points by the minimum requirements, and save the schemes in the $\mathrm{N} \times \mathrm{M}$ Array allocationMetrix.

Step 1.3 calculate the allocating amounts of every farmland operational points and save in the Array conflictResource,check if there is conflict in the pre-allocation scheme of the farm machinery $j$. If conflictResource[j] $>K_{j},\left(K_{j}\right.$ is the supplied amount of farm machinery $j$ ),then there is conflict and turn to Step2. Otherwise exit.

Step2. Generate strategies.

Step 2.1 If conflictResource $[j]>K_{j}$, then conflict $\operatorname{Count}[j]=$ conflictResource $[j]-K_{j}$. Otherwise, conflict Count $[j]=0$.

Step 2.2 If allocationMatrix $[i][j]>0$,

Step 2.2.1 If conflictCount $[j]>$ allocationMatrix $[i][j]$, the strategies amount of farmland $i$ strategyCount $[i]=$ allocationMatrix $[i][j]+1$,otherwise strategyCount $[i]=$ conflict Count $[j]+1$.

Step 2.2.2 If strategyCount $[i]>1$,calculate the cost of every strategy, strategyArray $[i]=\{0,1,2, \ldots$, strategy Count $[i]-1\}$, strategyCost $[i]=\{0 \times \operatorname{Cost}[j], 1 \times$ Cost $[j], \ldots,($ strategyCount $[i]-1) \times \operatorname{Cost}[j]\}$.

Step 2.3 The strategy array of every task is generated and then turn to Step3.

Step3. Calculate of payment matrix.

Step 3.1Calculate the total strategy combination amounts of tasks except task $i$, which is denoted as NumCols $[i]$. NumCols $[i]=$ strategyCount $[1] \times$ strategyCount $[2] \times \ldots \times$ strategyCount $[i-1] \times$ strategyCount $[i+1] \times \ldots \times$ strategyCount $[N]$.

Step 3.2 Create Array Payoff $[i]$ with strategyCount $[i] \times N u m C o l s[i]$, which is payment matrix of farmland $i$.

Step 3.3 Calculate the profit of every strategy combination and save in returnArray[NumCols[i]].

Step 3.4 Calculate the value of Payoff[i] and turn to Step4. Step4. Get the solution of Nash equilibrium.

Nash equilibrium is NP problem, Gambit is software and tool which can build and analyze finite extensive non-cooperative game strategy, the agricultural emergency allocation problem is a finite non-cooperative game problem.

\section{Experiments and Analysis}

To verify the effective of the two kinds of emergency scheduling and allocating algorithms proposed in the paper, the instances of farmland and farm machinery are produced randomly. Emergency Scheduling and Allocating Algorithm based on Priority Rules is denoted as $A L G_{l}$. Scheduling and Allocating Algorithm Based on the Non-Cooperative Game Theory is denoted as $A L G_{2}$. The number of tasks is 5 and that of resources is 4. The current date is June 12 and the deadline is June $18 . \mathrm{S}_{1}$ is $266.7 \mathrm{hm}^{2}, \mathrm{~S}_{2}$ is $466.7 \mathrm{hm}^{2}, \mathrm{~S}_{3}$ is $366.7 \mathrm{hm}^{2}, \mathrm{~S}_{4}$ is $413.3 \mathrm{hm}^{2}$ and $\mathrm{S}_{5}$ is $386.7 \mathrm{hm}^{2}$. The farmland grades are $1,1,2,2$ and 2 respectively. The service ability of farm machinery is $5 \mathrm{hm}^{2}$ per day. The response time of farm machinery is June $12^{\text {th }}$, June 14 th, June 13th and June $12^{\text {th }}$ respectively. The machinery amount is $10,35,25$ and 10.Table 1 is the temporal distance among emergency tasks and resources. 


\section{Table 1. The Temporal Distance among Emergency Tasks and Resources (Day)}

\begin{tabular}{|c|c|c|c|c|c|}
\hline No & $F_{1}$ & $F_{2}$ & $F_{3}$ & $F_{4}$ & $F_{5}$ \\
\hline$M_{1}$ & 0.3 & 0.2 & 0.2 & 0.3 & 0.1 \\
\hline$M_{2}$ & 0.4 & 0.1 & 0.4 & 0.4 & 0.2 \\
\hline$M_{3}$ & 0.5 & 0.2 & 0.5 & 0.6 & 0.2 \\
\hline$M_{4}$ & 0.1 & 0.4 & 0.5 & 0.1 & 0.3 \\
\hline
\end{tabular}

The scheduling scheme of $A L G_{1}$ is denoted as $S_{1}$ and that of $A L G_{2}$ is denoted as $S_{2}$. The comparison of the scheduling schemes from the aspects of scheduling cost and the allocating amounts with $A L G_{1}$ and $A L G_{2}$ is shown in table2.

Table 2. The Comparison between ALG1 and ALG2

\begin{tabular}{|c|c|c|c|c|c|c|c|}
\hline & Schemes & $F_{1}$ & $F_{2}$ & $F_{3}$ & $F_{4}$ & $F_{5}$ & Total \\
\hline $\begin{array}{c}\text { Scheduling } \\
\text { cost(yuan) }\end{array}$ & $S_{1}$ & 4,832 & 6,512 & 6,741 & 5,328 & 2,117 & 21,450 \\
\cline { 2 - 8 } & $S_{2}$ & 894 & 1,456 & 2,456 & 4,576 & 2,458 & 15,498 \\
\hline $\begin{array}{c}\text { Completion } \\
\text { time(day) }\end{array}$ & $S_{1}$ & 6.8 & 6.9 & 7 & 6.8 & 7 & 7 \\
\cline { 2 - 8 } & $S_{2}$ & 6.7 & 6.8 & 6.6 & 6.5 & 6.3 & 6.8 \\
\hline
\end{tabular}

The compared results from table 2 can be seen that the scheduling cost of $A L G_{l}$ is lower than that of $A L G_{2}$ and the allocating amounts of $A L G_{1}$ are less than that of $A L G_{2}$.In order to verify the effectiveness of $A L G_{2}$ in the paper, the further analysis and comparison are made and five sets of farmland instances are produced randomly. $s c_{l}$ means the scheduling cost of $A L G_{1}$ and $s c_{2}$ means that of $A L G_{2}$. $c t_{1}$ means the allocating amounts of $A L G_{1}$ and $c t_{2}$ means that of $A L G_{2}$. Result $t_{1}$ and Result $_{2}$, which are used to make comparisons at aspects of the scheduling cost and the allocating amounts ,can be defined as the following.

Table 3. The Comparison between ALG1 and ALG2

\begin{tabular}{|c|c|c|c|c|c|c|}
\hline \multirow{2}{*}{ NO } & \multicolumn{3}{|c|}{ Scheduling Cost(yuan) } & \multicolumn{3}{c|}{ Completion time(day) } \\
\cline { 2 - 7 } & $A L G_{I}$ & $A L G_{2}$ & Result $_{l}$ & $A L G_{l}$ & $A L G_{2}$ & Result $_{2}$ \\
\hline 1 & 23,743 & 19,856 & $16.37 \%$ & 9 & 8.8 & $2.22 \%$ \\
\hline 2 & 21,155 & 16,300 & $22.95 \%$ & 8 & 7.9 & $1.25 \%$ \\
\hline 3 & 19,537 & 14,657 & $24.98 \%$ & 7.9 & 7.6 & $3.80 \%$ \\
\hline 4 & 19,758 & 17,569 & $11.08 \%$ & 7.9 & 7.8 & $1.27 \%$ \\
\hline 5 & 20,369 & 17,943 & $11.91 \%$ & 8 & 7.8 & $2.50 \%$ \\
\hline
\end{tabular}

Therefore, it is concluded that the scheduling and allocating algorithm based on the non-cooperative game theory $\left(A L G_{2}\right)$ proposed in the paper is more effective and reasonable.

\section{Conclusions}

The characteristics of emergency scheduling and allocating are analyzed farm machinery emergency scheduling and allocating mode leading by management department is proposed and the optimization model with the goal of cost as low as possible and completing emergency tasks within the prescribed period is established in the paper. According to the characteristics of farm machinery emergency scheduling and 
allocating, the scheduling and allocating algorithm with priority rules and the scheduling and allocating algorithm based on the non-cooperative game theory are proposed. The problems of tasks selection based on priority and resource competition conflict are discussed. The effectiveness of the algorithm is verified through experiment in the paper. Currently, there are no sophisticated algorithms to solve the solution of equilibrium for Non-Cooperative Game problem. And the algorithm based on the Non-Cooperative Game Theory has strong dependence on the specific problems. Farm machinery emergency scheduling and allocating is complex and large system engineering, while the scheduling and allocating rules should be further studied in the future.

\section{Acknowledgment}

This work is partially supported by the Key Project Fund for science and technology research of Hebei Higher Education Institutions (Grant No. Z2014168) and the project of Baoding science and Technology Bureau(Grant No. 15ZR003). The authors also gratefully acknowledge the helpful comments and suggestions of the reviewers, which have improved the presentation.

\section{References}

[1] Fan Zhang, Guifa Teng,Shuhui Chang.Study on Farm machinery Scheduling and allocating problem with heuristic priority rules[J].ICIC Express Letters.vol.6,pp1797-1802,July 2012.

[2] Zefei Geng, Feihu Hu, Huimin Chen,Linyan Sun. Research on Multiple Disaster Places Emergency Resource scheduling and its implementation[J]. Computer Application and Software. vol.27, no.2,pp 63-66, 2010.

[3] BenArieh,D. and Chopra,M. Evolutionary Game-Theoretic Approach for Shop Floor Control[C].Proceedings of the IEEE International Conference on Systems, Man and Cybernetics, 1998(1):463-468.

[4] Yi Kan,Wang Ruchuan. Nash Equilibrium Based Task Scheduling Algorithm of Multi-schedulers in Grid Computing [J]. Acta Electronica Sinica, 2009, 37(2):91-95.

[5] Yang Jijun,Wu qidi,Cheng Yan,Xu Weisheng. Contingency Plans of Unconventional Emergency Based on Sequential Decision-making [J]. Journal of Tongji University (Natural Science), 2009, 37(2):91-95.

[6] Li Zhijie,Cheng Chuntian,Huang feixue,Li Xin. A Sequential Game-Based Resource Allocation Strategy in Grid Environment [J].Journal of Software. 2006, 17(11):157-167.

[7] Li Mingxin,Chen Shanzhi,Xie Dongliang,Hu Bo. Resource Allocation and Admission Control Based on Non-Cooperation Game in Heterogeneous Wireless Networks. [J].Journal of Software. 2010,21(8):259-271.

[8] The Study of the Router Internet Resource Management Based on Game Theory for Internet Survivability[D]. Xi'an Electronic and Science University,2006

[9] Wang Changjun,Xi Yugeng.Modeling and analysis of non-cooperative game with single machine scheduling[J], 2005(31),4:516-522.

[10] Liqun Chen. Review on the Research of Material Distribution in Emergency Logistics [J]. , Logistics Sci-Tech, no.12,2008.

[11] Jijun Yang, Weisheng Xu,Wujun Huang. Modeling and analyzing of simulation based on non-cooperative games for multiple emergency locations in resources scheduling [J]. Journal of Computer Applications, vol 28, no.6, pp1620-1623, 2008.

[12] Yingtao Chen. Optimization Models and Algorithms of Emergency Resource Dispatching under Time Constraints[D].University of Henan,2007.

[13] Chunlin Liu. The Study on Optimal Model for a Kind of Emergency Material Dispatch Problem[J]. Chinese Journal of Management Science, vol. 11, no.3.pp 29-36, 2001.

[14] Yi Zhang. Route choice for transporting exigency succor materials [J]. Journal of Safety and Environment, no.3.pp51-53, 2006.

[15] Zhiyun Zhou, Cheng Song. How to Select the Optimal Emergency Logistics Route Based on Grey Theory[J]. Logistics Technology, vol, 27, no.1,pp46-48,2008.

[16] Daoyuan Yu, Jian Huang. Application research of combinational rule heuristic algorithm in modul manufacture scheduling[J]. Application Research of Computers,vol.24,no.11,pp185-187,2007

[17] Jianming Zhu,Jun Huang, Degang Liu,Jiye Han. Randomized Algorithm for Vehicle Routing Model for Medical Supplies in Large-Scale Emergencies [J]. Operations Research and Management Science, Vol. 19, No. 1.pp 9-14, 2010

[18] Gambit: Software tools for game theory.Version:2010-09-01. http://www.gambit-project.org/doc/index.html. 


\section{Authors}

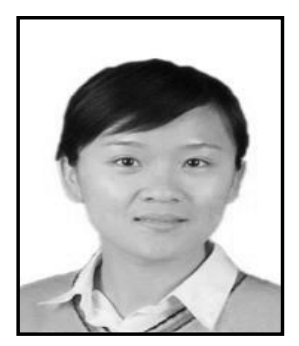

Fan Zhang is working as a lecturer at School of Information Science and Technology, Agricultural University of Hebei. I received $\mathrm{Ph} . \mathrm{D}$. degree in 2012 and the field of research is artificial intelligence applied technology.

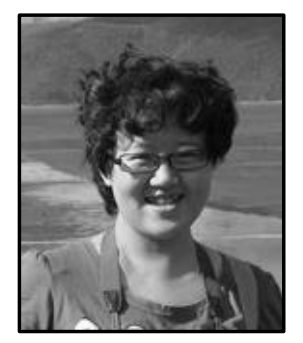

Yuan Gao is working as a lecturer at School of Information Science and Technology, Agricultural University of Hebei. Her field of research is artificial intelligence applied technology.

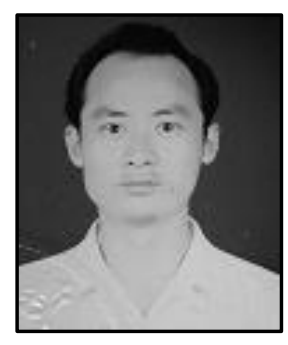

ChenChen is working as a lecturer at School of Information Science and Technology, Agricultural University of Hebei. His field of research is artificial intelligence applied technology. 